\title{
Linking Clustering Properties and the Evolution of Low Surface Brightness Galaxies
}

\author{
Dominik J. Bomans ${ }^{1}$, and S. Dominik Rosenbaum ${ }^{1,2}$ \\ ${ }^{1}$ Astronomical Institute, Ruhr-University Bochum, 44780 Bochum, Germany \\ email: bomans@astro.rub.de \\ ${ }^{2}$ DLR Oberpfaffenhofen, 82234 Weßling, Germany
}

\begin{abstract}
The reasons for the presence of two branches of galaxy evolution, one producing high surface brightness disks and one creating low surface brightness disks, is still unknown. Possible are the imprint of the properties of the dark matter halo, as well as evolutionary effects. In this paper we present an analysis of the clustering properties of LSB and HSB galaxies using the Sloan Digital Sky Survey. We show that LSB galaxies reside in regions of lower galaxy density than HSB galaxies on all scales between 0.8 and $8 \mathrm{Mpc}$, from scales of galaxy pairs to filaments of the Large Scale Structure. This implies a probable scenario of LSB galaxies preferentially forming as a result of local peaks in the large-scale valleys of the primordial density distribution.
\end{abstract}

Keywords. galaxies: formation, galaxies: evolution, galaxies: distances and redshifts, galaxies: fundamental parameters, galaxies: statistics, (cosmology:) large-scale structure of universe

\section{Introduction}

The presence of a significant population of Low Surface Brightness (LSB) galaxies in the local universe (Disney 1976) is by now well established (e.g., Impey \& Bothun 1997, Davies et al. 1997). Still the question of their origin and evolution is an unsettled issue. The basic properties of LSB galaxies is the central surface brightness of $\mu_{B}=22.5$ mag $(3 \sigma$ below the Freeman value (Freeman 1970)). Furthermore, they are mostly of blue color, have a high gas content, and low metallicity. All these global parameters indicate a less evolved stellar population compared to normal, high surface brightness (HSB) galaxies. It is important to emphasize here, that this is the definition of field LSB galaxies, which are dominated by disk galaxies and dwarf irregular galaxies. The LSB galaxy population in the very high density regions, galaxy clusters, is dominated by dwarf elliptical galaxies (e.g., Adami et al. 2006), which hints at a different formation mechanism (or different evolution). In this paper we will concentrate on field LSB galaxies. The global properties of field LSB galaxies do not directly show whether the galaxies formed stars at a later cosmic epoch, or if they had a generally depressed star formation starting in the early universe. Inhibiting star formation in LSB disks seems to be a natural consequence of the the low surface densities, which often stay below the Kennicutt criterion for star formation (Pickering et al. 1997), but this star formation criterion appears to be oversimplified (see Schaye, this conference). Analyses of integral colors and/or spectra show that the stellar population mix in LSB galaxies is different from HSB galaxies and that the age of the dominant stellar population in LSB galaxies tends to be younger compared to HSB galaxies (Bell et al. 2000, Haberzettl et al. 2007, and Haberzettl et al., this conference).

This does not yet answer the question what determines the decision of baryons in a dark matter (DM) halo to form or evolve into a LSB or HSB galaxy. It is a nature vs. nurture question. The properties of the DM halo may imprint a tendency to develop 
low surface brightness disks in case of a large halo spin parameter $\lambda$, as proposed by Dalcanton et al. (1997) and Boissier et al. (2003). Alternatively, the LSB galaxies could form preferentially in environments of low galaxy density and would therefore encounter less frequent galaxy interaction and mergers due to smaller number of neighbors. This missing stirring of the low surface density disks would prevent efficient star formation.

It is possible to test these ideas by investigating the clustering properties of LSB galaxies at low redshift. Up to now, there are only a few studies of the environment of LSB galaxies. Zaritsky \& Lorrimer (1993) and Bothun et al. (1993) showed that LSB galaxies have a deficit of neighboring galaxies on scales less than 0.5 Mpc. Later, Mo et al. (1994) demonstrated that the cross correlation amplitude of LSB galaxies is smaller than that of HSB galaxies on scales $r<2 h^{-1}$ Mpc. Using the Sloan Digital Sky survey (SDSS) Early Data Release, Rosenbaum \& Bomans (2004) reported that LSB galaxies reside in regions of lower galaxy density than HSB galaxies on scales up to $8 \mathrm{Mpc}$.

\section{Sample}

In order to analyze a large sample of LSB galaxies with well determined distances and coherent photometric quality, we decided to use the SDSS spectroscopic sample. Due to non-uniform sky coverage around the north galactic pole in the earlier data releases, we chose the equatorial region for our analysis.

The sample used for the present analysis was drawn from the SDSS DR4. To select LSB disk galaxies we have to estimate the central disk surface brightness, which is not directly provided by the SDSS photometry due to the possible presence of bulges. We derived the central disk surface brightness by fitting an exponential profile to the SDSS photometry of the outer annuli and extrapolated to the central disk as soon as the measured point started to deviate due to a steep bulge light profile. We tested this method successfully against more detailed $2 \mathrm{~d}$ fits performed on a small subsample of our target galaxies using the IRAF/STSDAS isophote and the Galfit (Peng et al. 2002) software packages. After converting the photometry in $\mathrm{g}$ and $\mathrm{r}$ to Johnson B band using the standard SDSS conversion relations (Smith et al. 2002) and correcting the central surface brightness for Tolman dimming, we could use the normal $\mu_{B}=22.5 \mathrm{mag}$ criterion to divide the galaxy sample into LSB and HSB galaxies.

To minimize the effects of local deviation from the Hubble flow, we required the galaxies to have a redshift $z>0.05$. In order to limit the necessary corrections for Tolman dimming and for incompleteness, we further decided on a maximum redshift of $z<0.1$ for our sample. For further tests of the selection biases we split the final sample in two symmetric bins, $0.01<z<0.055$ and $0.055<z<0.1$. Disappointingly, the LSB contribution to the galaxy sample is small, just $0.65 \%$ (1141 LSB galaxies and 210702 HSB galaxies) and smaller than expected by scaling from the EDR. Apparently the SDSS spectroscopic follow-up has a severe selection bias against picking LSB galaxies, which are however present in the SDSS imaging material.

When plotting the luminosity distribution of the the LSB galaxies in the two redshift regimes, another interesting effect becomes visible, see Fig. 1. Apparently, the lower redshift sample is dominated by lower luminosity galaxies (roughly comparable to the $\mathrm{SMC}$ ) than the higher redshift sample, where the typical luminosity is more like that of the LMC. Due to the selection function of the SDSS spectroscopic sample we encounter a Malmquist bias. We therefore have to keep in mind for the following discussion that we investigate samples of galaxies with quite different luminosities (and presumably masses) in the the low and the higher redshift subsamples. 

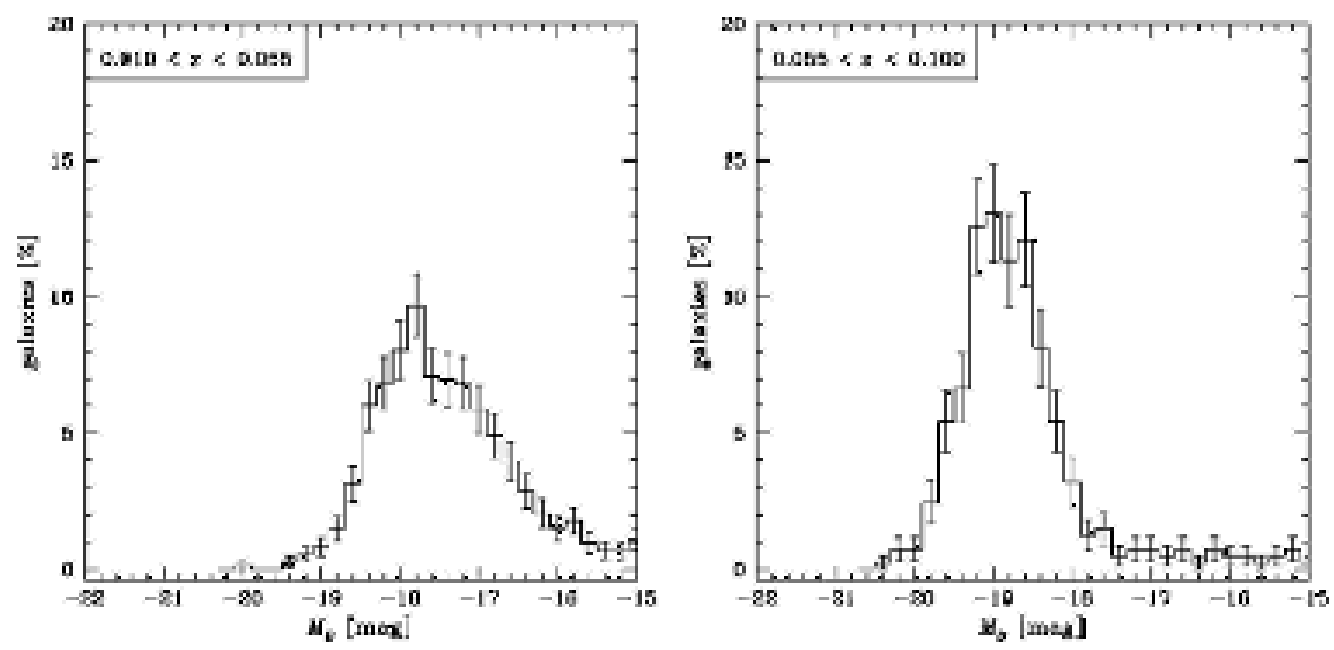

Figure 1. B-Band luminosity distribution of our SDSS DR4 based LSB galaxy sample. The left panel shows the low redshift bin, the right panel the high redshift bin. Note the significantly different populations in the two panels due to the selection function of the SDSS spectroscopic sample.
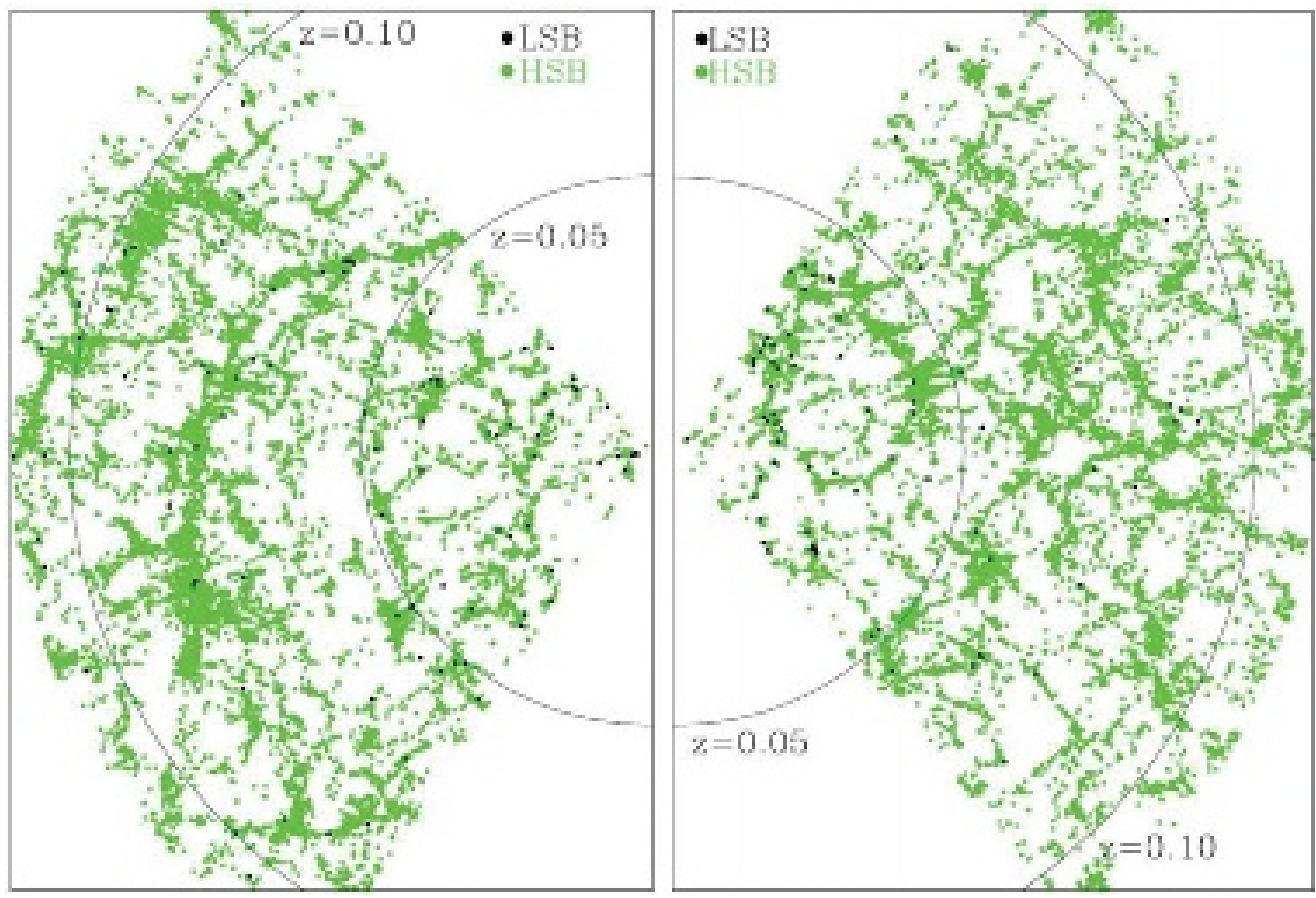

Figure 2. Two $2.5 \mathrm{deg}$ thick pie slices of the analyzed SDSS data. The HSB galaxies are plotted as light gray, the LSB galaxies as black dots. Note the LSB galaxies in the low density regions.

\section{Results and conclusions}

Plotting the galaxies in the pie slice diagrams already showed the apparent tendency of the LSB galaxies to follow the large scale structure (LSS) defined by the HSB galaxies, but populated preferentially the outer rims of the filaments, not their centers, see Fig. 2 . 

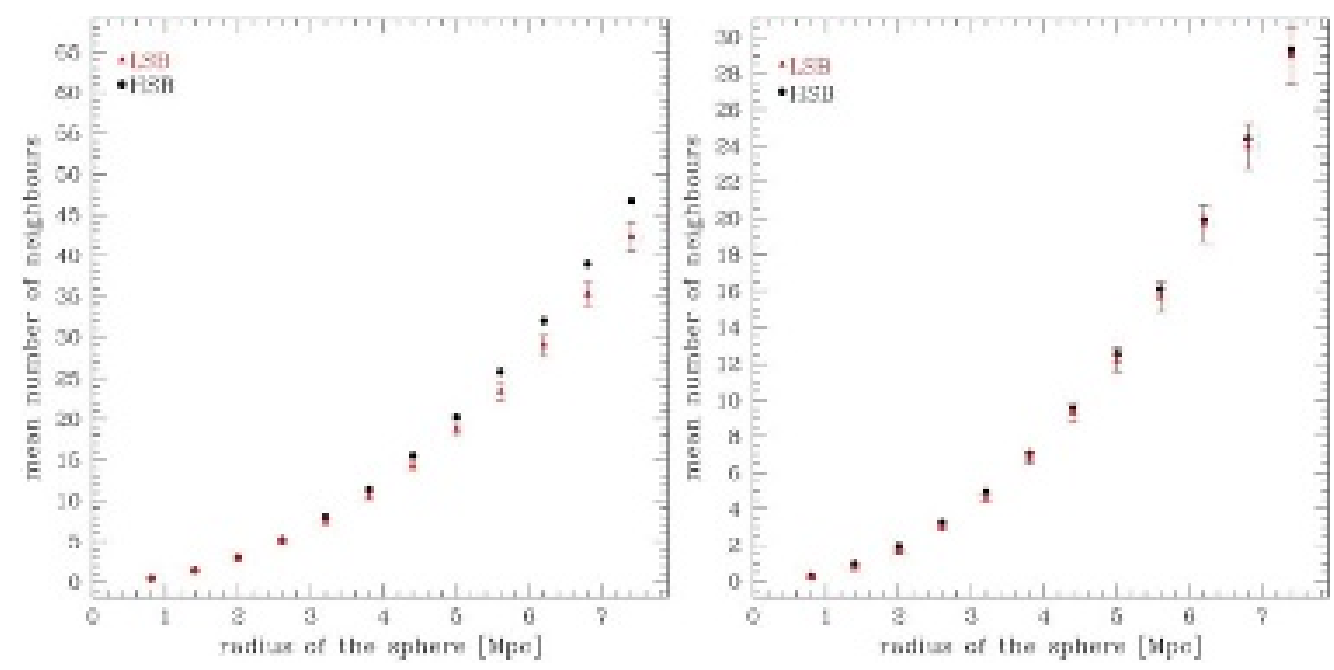

Figure 3. Results of the neighbor count analysis in the cluster corrected final sample, for the low redshift sample (left panel) and the high redshift sample (right panel). The HSB galaxy neighbor counts are plotted as black dots, the LSB galaxy neighbor counts as gray triangles. Error bars give the statistical uncertainties.

In order to keep our sample a field LSB galaxies sample, we performed a simple galaxy cluster search based in the characteristic radial finger-like appearance of bound systems in pie slice plots on the data set. We tested the results against the C4 cluster search algorithm (Miller et al. 2005) and were able to recover all galaxies clusters found with the C4 method. Using our code we then defined regions around each cluster to clean the sample from high probability cluster member galaxies.

Using these two fully corrected galaxy samples we calculated number counts in spheres of increasing radii (in Mpc) for both LSB galaxy subsamples and the corresponding HSB galaxy subsamples. The results are plotted in Fig. 3. In the low redshift subsample the LSB galaxies have a lower number of neighbors than the HSB galaxies in all radial bins up to the investigated limit of $r=8 \mathrm{Mpc}$. This effect is formally significant at all radii $r>2.6$ Mpc. In the higher redshift subsample the same pattern is present, too, but with a lower statistical significance. Here the LSB galaxies have a formally significant lower number of neighbors in the interval $2<r<3.8 \mathrm{Mpc}$. The difference in the two redshift bins is most probably not a population effect, but simply due to poor statistics especially in the high redshift subsample. There are only 200 LSB galaxies (and 69000 HSB galaxies) in the high redshift sample, while there are 400 LSB and 31000 HSB galaxies in the final low redshift sample.

While not formally significant in all radial bins investigated in our study, the trend seems to be clear: LSB galaxies reside in regions of lower galaxy density than HSB galaxies, from the smallest scales up to the size scale of groups and filaments of the LSS. This is qualitatively the same result we obtained in our pilot study (Rosenbaum et al. 2004). This implies the following scenario: LSB galaxies form at local peaks in the lower density part of the primordial density fluctuations, meaning more inside future voids, and then drift into the filaments in relatively recent times. The presence, but relative rare occurrence, of LSB galaxies in voids (see Fig. 2) can be taken as a natural result in this picture.

This simple model is fully consistent with all data and makes a couple of testable predictions: There should be a (small) enhancement of star formation density of the 
universe at redshift $z<1$ due to LSB galaxies reaching the filaments in the LSS. It is not clear if this effect can be detected, since an enhanced star formation rate in LSB galaxies would still result in a relatively low total star formation rate per LSB galaxy. This may completely be swamped by the star formation density of the HSB galaxies. Another effect should be more traceable: The LSB galaxies farther inside the filaments should exhibit on average a higher recent star formation rate than the LSB galaxies in and near voids. Due to the large extent of the LSB galaxies with $z<0.1$ the SDSS spectra are not directly usable for such a test. The small SDSS fiber, which is always centered on the brightest spot of the galaxy (mostly the center) would introduce a bias against star formation in the large disks of LSB galaxies. A follow-up program to test the prediction by narrow band imaging is underway.

\section{Acknowledgements}

This research was supported by the Deutsche Forschungsgemeinschaft project GRK 787 (the Bochum-Bonn Graduate School 'Galaxy Groups as Laboratories for Baryonic and Dark Matter'). We thank L. Haberzettl, C Trachternach, and R.-J. Dettmar for discussions and $\mathrm{K}$. Weis for comments on the manuscript.

Funding for the SDSS and SDSS-II has been provided by the Alfred P. Sloan Foundation, the Participating Institutions, the National Science Foundation, the U.S. Department of Energy, the National Aeronautics and Space Administration, the Japanese Monbukagakusho, the Max Planck Society, and the Higher Education Funding Council for England. The SDSS Web Site is http://www.sdss.org/. The SDSS is managed by the Astrophysical Research Consortium for the Participating Institutions.

\section{References}

Adami, C., Scheidegger, R., Ulmer, M., Durret, F., Mazure, A., West, M. J., Conselice, C.J., Gregg, M., Kasun, S., Pelló, R., \& Picat, J.P. 2006, A $ళ$ A 459, 679

Bell, E. F., Barnaby, D., Bower, R. G., de Jong, R. S., Harper, D. A., Hereld, M., Loewenstein, R. F., \& Rauscher, B. J. 2000, MNRAS 312, 470

Boissier, S., Monnier Ragaigne, D., Prantzos, N., van Driel, W., Balkowski, C., O'Neil, K. 2003, MNRAS 343, 653

Bothun, G. D., Schombert, J. M., Impey, C. D., Sprayberry, D., \& McGaugh, S. S. 1993, AJ 106,530

Davies, J. I., Impey, C. A., \& Phillipps, S. 1997, The Low Surface Brightness Universe, IAU Symposium 170

Dalcanton, J. J., Spergel, D. N., \& Summers, F. J. 1997, ApJ 482, 659

Disney, M. J. 1976, Nature 263, 573

Freeman, K. C. 1970, ApJ 160, 811

Haberzettl, L., Bomans, D. J., \& Dettmar, R.-J. 2007, A\&SA, in press, (astro-ph/0707.0492)

Impey, C. D., \& Bothun, G. D. 1997, ARAA 35, 267

Miller, C. J., et 17 alii 2005, $A J$ 130, 968

Mo, H. J., McGaugh, S. S., \& Bothun, G. D. 1994, MNRAS 295, 319

Peng, C. Y., Ho, L. C., Impey, C. D., \& Rix, H.-W. 2002, AJ 124, 266

Pickering, T. E., Impey, C. D., van Gorkom, J. H., \& Bothun, G. D. 1997, AJ 114, 1858

Rosenbaum, S. D., \& Bomans, D. J. 2004, A 6 A 422, L5

Smith, J. A. et 21 alii $2002, A J 123,2121$

Zaritsky, D., \& Lorrimer, S. J. 1993, The Evolution of Galaxies and Their Environment, NASA Ames Research Center, p. 82 